\title{
Precision HEOR: A Natural Complement to Precision Medicine
}

\section{Yixi Chen*, Chengming Gu and Peng Dong}

Pfizer Investment Co. Ltd, Beijing, China

Journal of Personalized Medicine (JPM) has recently reported a new concept in the field of Health Economics and Outcomes Research (HEOR), namely, Precision HEOR [1]. We hold the belief that precision HEOR is a natural complement to precision medicine, with an emphasis on weighing costs while looking for customized treatment for individual patient with best available clinical outcomes. Looking back, the field of HEOR has emerged to support allocating limited healthcare resources as efficiently as possible in face of an ever-increasing demand in healthcare. Benefiting from big data, precision HEOR is bringing traditional HEOR to the next level at which patient population is precisely stratified, economic models are precisely constructed, uncertainties are precisely handled, and recommendations for healthcare decision making are precisely made. Apart from the many perspectives of precision HEOR in different health sector decision contexts described in the JPM paper, I envision two starting applications where precision HEOR holds promise for increasing healthcare efficiency.

\section{Clinical Decision Support System (CDSS)}

The CDSSs I refer to are those using machine learning to help physicians with decision-making tasks, e.g. giving treatment recommendations predicted to yield the best clinical outcomes to a specific patient. This has particular merit in assisting less-experienced physicians to make clinical decisions as sound as possible, or in serving as another layer of evidence for experienced physicians to confirm or polish their decisions. However, the drawback of this type of CDSSs is that medical costs are easily overlooked, yet they are becoming an important factor in a physician's decision making process under the pressure of cost containment. Precision HEOR will be able to make economic evaluations to each recommendation, whether it is a simple prescription or a more complicated treatment approach, before the optimal one is presented to the user interface of a CDSS. It is important to note that there is no difference between precision HEOR and traditional HEOR in selecting data input for the economic evaluation - all relevant cost components (including direct and indirect costs) and appropriate patient outcomes measures (e.g. different clinical endpoints, patient-reported outcomes) should be included. Therefore, the CDSSgenerated recommendations ensure best value (health benefit for every dollar spent) given evidence and healthcare resource limitations. In addition, CDSSs might reduce information asymmetry and accelerate the patient-physician shared decision making by presenting to patients simple and transparent data of the effectiveness and costs of different treatment approaches proposed by their physicians.

\section{Precision Reimbursement Decision Making}

Payers, another key healthcare stakeholder, will also be highly likely to utilize precision HEOR in making reimbursement decisions with a higher degree of precision. The one-size-fits-all reimbursement may risk wasting healthcare resources due to supply induced demand in patients with relatively low risk and/or severity of a certain disease, and underreimbursement for those who need to be prioritized for more intensive treatment. In 2015, the Chinese government started to explicitly require precision in serious illness insurance [2]. We argue that big-data-based risk prediction is the start for precision reimbursement. For example, our current efforts in constructing a risk prediction model for Chinese patients with atrial fibrillation proved to achieve better performance than the Framingham model (with AUROC increased from 0.655 to 0.744, and AUPRC increased from 0.175 to 0.243) [3]. Based on the multiple treatment options and outcomes forecasted by this model, precision HEOR will evaluate high-precision economic impacts of disease prevention or early intervention in order to provide evidence to support more precise reimbursement decision making in terms of differentiated reimbursement levels to different population stratified by risk of diseases.

Similar to precision medicine, precision HEOR is big-data-driven and thus requires multi-disciplinary collaborations throughout a study, from identifying relevant research questions and selecting targeted patient population, to precise stratification of these patients and feature selection, to building predictive and/or economic models embedded with calculation algorithms, and finally presenting modeling results and extrapolating them for the greater population. It is therefore necessary to gather collective intelligence from experts in HEOR, medicine and data science in order to conduct precision HEOR. Specifically, what distinguishes precision HEOR from traditional HEOR is the incorporation of two major data science methodologies, namely, predictive modeling and patient similarity analysis (also called precision cohort analysis); while the former ensures good representations of the probability of different outcomes serving as a ground for good decisions, the latter provides a way to find clinically similar patient to a certain index patient for decision support and for comparative effectiveness and costs [4]. Many interesting and stateof-the-art techniques can be used to achieve these goals but they are beyond this short communication's scope to address. We are currently working closely with brilliant clinicians and data scientists on the first precision HEOR case in the cardiovascular area and we hope to disclose the results in the near future. As stated in the JPM paper, "the combined hopes for precision medicine and precision HEOR are not only that individual patients receive the best possible medical care for their situation, but also that overall societal healthcare resource allocation will achieve the best outcomes for the money spent [1]".

\section{References}

1. Chen Y, Guzauskas G, Gu C, Wang B, Furnback W, et al. (2016) Precision health economics and outcomes research to support precision medicine: Big data meets patient heterogeneity on the road to value. J Pers Med 6: 20.

2. http://www.gov.cn/zhengce/content/2015-08/02/content_10041.htm

3. Li X, Xie G, Guo S, Xu M, Xie X (2016) AMIA.

4. Ghahramani $Z$ (2015) Probabilistic machine learning and artificial intelligence Nature 521: 452-459.

*Corresponding author: Yixi Chen, Pfizer Investment Co. Ltd, Beijing, China, Tel: +86 15910977334; E-mail: YiXi.Chen@pfizer.com

Received December 01, 2016; Accepted January 07, 2017; Published January 13, 2017

Citation: Chen Y, Gu C, Dong P (2017) Precision HEOR: A Natural Complement to Precision Medicine. Health Care Current Reviews 5: 182. doi: 10.4172/23754273.1000182

Copyright: ( 2017 Chen Y, et al. This is an open-access article distributed under the terms of the Creative Commons Attribution License, which permits unrestricted use, distribution, and reproduction in any medium, provided the original author and source are credited. 